Research Square

\title{
Taylor expansion based stereo image reversible data hiding
}

Xinyang Ying ( $\nabla$ yingxinyang@nbu.edu.cn )

Ningbo University

Guobing Zhou

Ningbo University

Research

Keywords: Stereo Image Reversible Data Hiding, Taylor expansion (TE), Histogram shifting, Multi-level hiding.

Posted Date: October 2nd, 2020

DOI: https://doi.org/10.21203/rs.3.rs-85033/v1

License: (1) This work is licensed under a Creative Commons Attribution 4.0 International License. Read Full License 


\title{
Taylor expansion based stereo image reversible data hiding
}

\author{
Xinyang Ying* Guobing Zhou
}

\begin{abstract}
The reversible data hiding allows original image to be completely recovered from the stego image when the secret data has been extracted, it is has drawn a lot of attentions from researchers. In this paper, a novel Taylor Expansion (TE) based stereo image reversible data hiding method is presented. Since the prediction accuracy is essential to the data hiding performance, a novel TE based predictor using correlations of two views of the stereo image is proposed. TE can fully exploit strong relationships between matched pixels in the stereo image so that the accuracy of the prediction can be improved. Then, histogram shifting is utilized to embed data to decrease distortion of stereo images, and multi-level hiding can increase embedding capacity. Experimental results show that the proposed method is superior to some existing data hiding methods considering embedding capacity and the quality of the stego stereo images.
\end{abstract}

Keywords: Stereo Image Reversible Data Hiding; Taylor expansion (TE); Histogram shifting; Multi-level hiding.

\section{Introduction}

In recent years, the stereo image as the main representations of the 3D image has drawn much attention, since it can give people a realistic vision [1-3]. Due to the development of the multimedia technology, people can easily modify the stereo image and security problems may be raised. The data hiding method can solves this kind of problem, but in general it will change the image content a little although the visual quality is not decreased much [4-5]. However, for military, judicial and medical images, any tiny modification is not allowed. The reversible data hiding method can restore the original image when the secret data is extracted, which can be applied in these above special applications [6-7].

The reversible data hiding method can be divided into three types. The first type is to leave vacant space for embedding data by losslessly compressing the least significant bit (LSB) plane of the image [8]. However, due to the low compression ratio, this kind of the data hiding method cannot obtain the high embedding capacity with low image quality. The second type is based on difference expansion (DE). Tian computed the difference between two neighbor pixels, and then expended it for embedding the secret data [9]. However, in the edge region or complex texture region, the differences between two adjacent pixels are large, which will lead to the distortion of the image. Alattar improved Tian's method, and it used generalized integer transform to increase embedding capacity [10]. Moreover, some other techniques were presented to improve the embedding performance, such as location map reduction [11-12], the prediction error instead of pixel difference [13-14], etc.

The third type is the histogram shifting (HS) based reversible data hiding method, which can improve quality and embedding capacity compared with the DE based reversible data hiding method. Ni et al. constructed a pixel histogram, and then emptied one bin nearby the peak by

*Correspondence: yxyemail@126.com

College of Science and Technology, Ningbo University, Wenwei Road, Ningbo, China shifting histograms between zero and peak [15]. The peak bin is utilized to embed data and its height determines the embedding capacity and the image quality. However, pixel histograms are often mean distributions and the peak point is not high enough, and it leads to the limitation of the image quality and the embedding capacity. In order to improve the embedding performance, sub-sampled images are computed by dividing the image, and the center pixel of each sub-sampled image is chosen as the reference pixel to calculate differences [16]. The corresponding difference histogram is Gaussian distribution, and the peak is higher compared with the pixel histogram. However, the embedding capacity can be still improved, because the differences between the reference pixel and the current pixel are still large for the complex texture areas. The median instead of the center pixel as the reference pixel is used to build the difference histogram so that the embedding performance is improved [17]. An et al. built statistical quantity histogram for reversibly data embedding based on clustering [18]. The method was robust to compression and random noise. Since the prediction value is similar to the current pixel, the prediction error histogram is superior to the difference histogram for data hiding. Thus, different kinds of predictors were presented to increase the prediction accuracy for obtaining the histogram with many bins around the bin zero, such as such as linear prediction [19], Median edge detector (MED) [20], rhombus prediction [21] and partial differential equation (PDE) [22]. Above predictors only employ intra-relationships to compute the predicted values for the current pixel. However, for the stereo image, besides intra-relationships, inter-relationships can be also used to improve the prediction.

The stereo image includes left and right views, which are captured for the same scene and have strong inter-relationships. The inter-view correlations of the stereo image can increase the embedding performance of the stereo image data hiding method. Yu et al. employed block matching to increase embedding capacity so that the capabilities of tamper detection and recovery were increased [23]. Zhou et al. computed the disparity of the 
stereo image and generated the disparity zero-watermark to protect the copyright of the stereo image, which is robust to common image processing [24]. Wang et al. used Ternary radial harmonic Fourier moments to obtain the inter-correlations for building the zero-watermark, which had the robustness [25]. But above stereo image data hiding methods are not reversible, stereo image reversible data hiding methods should be studied for the secret data transmission in the special area. Yang et al. presented DCT based stereo image reversible data hiding by using matched blocks [26], but the embedding capacity is not large. Luo et al. proposed disparity based stereo image reversible data hiding, which used the matched pixel to predict the current pixel for obtaining the prediction error histogram [27]. However, the difference between a pair of matched pixels is sometimes large, since they are captured by the different lighting.

In this paper, a novel stereo image reversible data hiding method based Taylor Expansion (TE) is presented. Since the prediction accuracy is very important for the data hiding performance, a novel TE based predictor employing correlations of two views in the stereo image is proposed. TE can fully exploit strong relationships between matched pixels in the stereo image so that it can increase the prediction accuracy of the stereo image. Secondly, histogram shifting is utilized to embed the secret data to degrade the distortion of the stereo image. Moreover, multi-level hiding can increase embedding capacity. Experimental results show that the proposed method is superior to some existing data hiding methods considering embedding capacity and the quality of the stego stereo images.

The remainder of this paper is organized as follows. Section 2 describes the proposed method based on Taylor Expansion. Section 3 proposes experimental results and discussions. Finally Section 4 gives the conclusion.

\section{The proposed stereo image RDH method based on Taylor Expansion}

In this section, at first the predictor based Taylor Expansion (TE) is designed to compute accurate prediction. Secondly, data hiding processes based on histogram shifting are given. Finally, data extracting processes are described in detail.

\subsection{Predictor based on TE (PTE)}

At first, the intra-relationships based predictor rhombus prediction is described. Then, a TE based predictor with the inter-view relationship is designed for predicting pixels in stereo images.

Since the prediction accuracy is essential to the data embedding performance, many predictors were proposed in many literatures. The rhombus prediction is the classical one by using intra-relationships. Let $f(x, y)$ be the pixel locating at $(x, y)$, and its eight neighbor pixels are shown in Fig. 1. For rhombus prediction, pixels are divided into rectangle and circle pixels as illustrated in Fig. 2. In rhombus prediction, the mean of four neighbor pixels is computed for the prediction value of the current pixel.

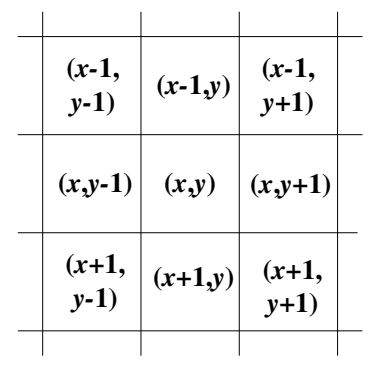

Fig. 1 Location of neighbor pixels

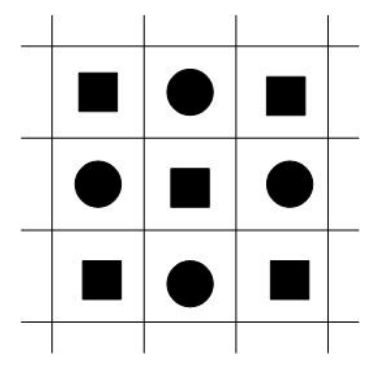

Fig. 2 Pixel pattern

$$
\hat{f}(x, y)=\left\lfloor\frac{\left(f_{1}+f_{2}+f_{3}+f_{4}\right)}{4}\right\rfloor
$$

where $f_{1}, f_{2}, f_{3}$ and $f_{4}$ are $f(x, y-1), f(x, y+1), f(x-1, y)$ and $f(x+1, y)$, respectively. However, for the stereo image, inter-correlations are sometimes closer than intra-correlations. In this section, a predictor based on Taylor Expansion (PTE) is designed to predict pixels in the stereo image by fully using inter-relationships of the stereo image.

Let $f^{L}(x, y)$ and $f^{R}(x, y)$ denote the left view pixel and the right view pixel, respectively. $f^{L}(x+h, y)$ and $f^{L}(x-h, y)$ are neighbor pixels of $f^{L}(x, y)$, which can be expanded as Eq. (2) by using the Second-order TE, where $h$ is the positive integer value.

$$
\begin{aligned}
& f^{L}(x+h, y) \approx f^{L}(x, y)+\frac{\partial f^{L}(x, y)}{\partial x} h+\frac{\partial^{2} f^{L}(x, y)}{2 ! \partial^{2} x} h^{2} \\
& f^{L}(x-h, y) \approx f^{L}(x, y)+\frac{\partial f^{L}(x, y)}{\partial x}(-h)+\frac{\partial^{2} f^{L}(x, y)}{2 ! \partial^{2} x} h^{2}
\end{aligned}
$$

The Eq. (2) can be changed as

$$
\begin{aligned}
& f^{L}(x+h, y)+f^{L}(x-h, y) \approx 2 f^{L}(x, y)+2 \frac{\partial^{2} f^{L}(x, y)}{2 ! \partial^{2} x} h^{2} \\
& f^{L}(x, y) \approx \frac{f^{L}(x+h, y)+f^{L}(x-h, y)}{2}-\frac{\partial^{2} f^{L}(x, y)}{2 ! \partial^{2} x} h^{2}
\end{aligned}
$$

where $\frac{\partial^{2} f(x, y)}{\partial^{2} x}$ is the Second-order partial derivative difference, and defined as 
$\frac{\partial^{2} f^{L}(x, y)}{\partial^{2} x}=f^{4}(x-h, y)+f^{4}(x+h, y)-2 f^{4}(x, y)$

Suppose $f^{L}(x, y)$ and $f^{R}(x, y-d)$ are matched pixels with the disparity value $d$, and they are have similarities. Thus, $\frac{\partial^{2} f(x, y)}{\partial^{2} x}$ can be nearly expressed as

$\frac{\partial^{2} f^{4}(x, y)}{\partial^{2} x} \approx f^{R}(x-h, y-d)+f^{R}(x+h, y-d)-2 f^{R}(x, y-d)$

When $h$ is smaller, the corresponding neighbor pixels are closer to the current pixel. Thus, we set $h$ to 1 , and based on Eqs.(3) and (5), $f^{L}(x, y)$ can be computed as

$$
\begin{aligned}
f^{L}(x, y) & \approx \frac{f^{L}(x+1, y)+f^{L}(x-1, y)}{2}- \\
& \frac{f^{R}(x-1, y-d)+f^{R}(x+1, y-d)-2 f^{R}(x, y-d)}{2}
\end{aligned}
$$

From Eq. (6), if the current pixel in the left view locates at the horizontal texture region, its prediction value is computed as

$$
\begin{aligned}
f^{L}(x, y)= & \frac{f^{L}(x+1, y)+f^{4}(x-1, y)}{2}- \\
& \frac{f^{R}(x-1, y-d)+f^{R}(x+1, y-d)-2 f^{R}(x, y-d)}{2}
\end{aligned}
$$

Similarly, if the current pixel locates at the vertical texture region, its predicted value is calculated as

$$
\begin{aligned}
\hat{f}^{L}(x, y)= & \frac{f^{L}(x, y+1)+f^{L}(x, y-1)}{2}- \\
& \frac{f^{\mathcal{R}}(x, y-1-d)+f^{\mathcal{R}}(x, y+1-d)-2 f^{\mathcal{A}}(x, y-d)}{2}
\end{aligned}
$$

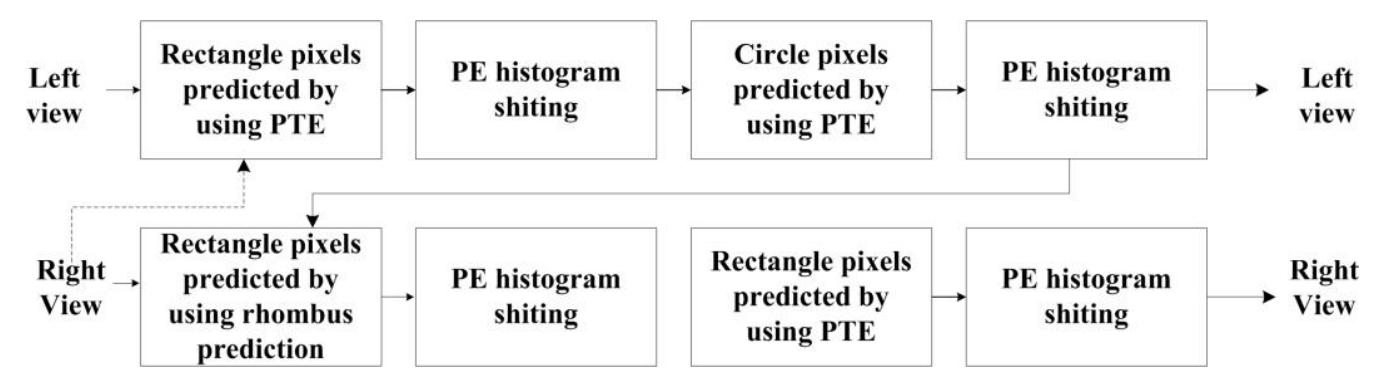

Fig. 3 Block diagram of data embedding

The main steps of reversible data hiding are listed as follows.

Step a-1. Pixels are classified into rectangle and circle pixels. Rectangle pixels are predicted temporarily by computing the mean of four neighbor circle pixels. Those predicted pixels and circle pixels form the temporary left view, and a disparity map is computed by using the graph-based global algorithm [28], denoted as $\boldsymbol{D}$.

Step a-2. Pixels in the left view are predicted by using PTE. At first, texture of the pixel is estimated according to Eq. (10).

where $T$ is the parameter to control the image texture estimation, and $H T, V T, S T$ and $C T$ represent horizontal texture, vertical texture, smooth texture and complex texture, respectively.
If the pixel locates at the smooth region, the pixel is predicted as

$$
\begin{aligned}
f^{L}(x, y)= & \frac{f^{4}(x, y+1)+f^{4}(x, y-1)+f^{4}(x+1, y)+f^{4}(x-1, y)}{4}- \\
& \frac{f^{R}(x, y-1-d)+f^{R}(x, y+1-d)-2 f^{R}(x, y-d)}{2}- \\
& \frac{f^{R}(x-1, y-d)+f^{R}(x+1, y-d)-2 f^{R}(x, y-d)}{2}
\end{aligned}
$$

\subsection{Processes of data hiding}

In the proposed stereo image data hiding method, pixels in the left view are predicted by using PTE. However, relationships of the left and right views are not strong any more after data embedding in the left view pixels, since the left view is modified. Thus, TE based predictor is not good at predicting for pixels of the right view, and instead rhombus prediction is used to predict pixels in the right view for data embedding. Pixels of each view are classified into circle and rectangle pixels as illustrated in Fig. 2. At first, rectangle pixels in the left view are predicted by using PTE, and then the secret data is embedded by using histogram shifting. Secondly, data is embedded in the circle pixels in the left view by using PTE and histogram shifting. Then, the secret data is embedded in the rectangle pixels of the right view, where the pixel is predicted by using rhombus prediction. Finally, the secret data is embedded into the circle pixels of the right view. The block diagram of data embedding is illustrated in Fig. 3.

$$
X^{L}=\left\{\begin{array}{l}
H T \text { if }\left|f{ }_{1}-f_{2}{ }_{2}\right| \leq T \\
V T \text { if }\left|f{ }_{3}-f{ }_{4}\right| \leq T \\
S T \text { if }\left|f{ }_{1}{ }_{1}-f{ }_{2}{ }_{2}\right| \leq T \&\left|f{ }_{3}-f{ }_{4}\right| \leq T \\
C T \text { ot herwise }
\end{array}\right.
$$

If $X^{L}$ is $H T$, Eq. (7) is used to compute the prediction value of $f^{L}(x, y)$. If $X^{L}$ is $V T$ or $S T$, Eq. (8) or Eq. (9) is utilized to compute the prediction value. If $X^{L}$ is $C T$, it is difficult to predict the current pixel, and the corresponding pixel is not used to embed data.

Step a-3. The corresponding prediction error $e$ is computed by

$$
e=f^{L}(x, y)-f^{L}(x, y)
$$


The prediction error histogram $\mathrm{H}=\{e\}$ with corresponding bins $\left\{\ldots b_{-2}, b_{-1}, b_{0}, b_{1}, b_{2} \ldots\right\}$ is built and obtained.

Step a-4. Empty bins by scanning $e$. Bins in the ranges of $[D L+1,2 \times D L+1]$ and $[-2 \times D L-1,-D L-1]$ are emptied without data embedding, where $D L$ is the embedding level. Corresponding $e$ is update as

$$
e^{\prime}=\left\{\begin{array}{l}
e+(a+1) \text { if } e \geq a+1 \\
e-(a+1) \text { if } e \leq-(D+1)
\end{array}\right.
$$

Specifically, if $e$ is equal or greater than $D L+1$, those bins are shifted to the right siede. When $e$ is equal or less than $-(D L+1)$, those bins are shifted to the other side.

Step a-5. The remaining bins are used to embed the secret data. Bins are in the range of $[-D L, D L]$ are shifted to embed $w$, where $w=\{0,1\}$ is the secret bit. When the absolute value of $e$ is equal to $D L, w$ is embedded, where $D L$ is decreased to 0 by step -1 . When $D L>0, w$ is embedded by using Eq. (13).

$$
e^{\prime}=\left\{\begin{array}{l}
e-(a+1) \text { if } e=-D, w=1 \\
e+(a+1) \text { if } e=a, w=1 \\
e-a \quad \text { if } e=-a, w=0 \\
e+a \quad \text { if } e=a, w=0
\end{array}\right.
$$

If $D L=0, w$ is embedded by using Eq. (14).

$$
e^{\prime}=\left\{\begin{array}{l}
e+1 \text { if } e=0, w=1 \\
e \quad \text { if } e=0, w=0
\end{array}\right.
$$

Step a-6. At that time, $b_{-1}$ is empty, and $e^{\prime}$ is scanned again to embed $w$.

$$
e^{\prime \prime}=\left\{\begin{array}{l}
e^{\prime}-1 \text { if } e^{\prime}=0, w=1 \\
e^{\prime} \text { if } e^{\prime}=0, w=0 \\
e^{\prime} \text { ot her } w \text { se }
\end{array}\right.
$$

Step a-7. All bins of the histogram are shifted, and pixels are modified as

$$
f^{L^{\prime}}(x, y)=\hat{f}^{L}(x, y)-e^{\prime \prime}
$$

Step a-8. The circle pixels of the left view are predicted by using PTE, and the secret data is embedded by using steps from a-3 to a-7, and the embedding process is the same as embedding data in the rectangle pixels.

Step a-9. Rhombus prediction is used to predict the rectangle pixels in the right view. Steps from a-3 to $\mathbf{a - 7}$ are operated to embed data into the rectangle pixels. Then, the secret data is embedded in the circle pixels.

Step a-10. The stego stereo image is computed finally.

From above steps, we can see that the embedding capacity and image quality are controlled by the embedding level $D L$. If $D L$ is decreased, the embedding capacity is decreased and the image quality is increased, and vice versa.

Since the pixel is in the range of $[0,255]$, during histogram shifting, overflow/underflow may be caused. When pixels are out of $[0,255]$ after shifting, they are not shifted and their locations are recorded. These locations are stored in the overhead information. Furthermore, the value of $D L$ is stored in overhead information as well. The least significant bit (LSB) replacement is used to embed overhead information in the four side regions of each view.

\subsection{Processes of data extraction}

At the receiving side, the secret data $\mathrm{W}=\{\mathrm{w}\}$ is extracted losslessly from the untampered stego stereo image. At first, $D L$ is extracted from the overhead information, and then the right view is recovered. Finally, the left view is restored. The main steps of data extraction are depicted in detail as follows.

Step b-1. Predict circle pixels in the right view by using the rhombus prediction.

Step b-2. Prediction error $e^{\prime \prime}$ is computed as

$$
e=\hat{f}^{L}(x, y)-f^{L^{\prime}}(x, y)
$$

Step b-3. Scan $e^{\prime \prime}$ to extract $w$ as

$$
w=\left\{\begin{array}{lll}
1 & \text { if } & e^{\prime \prime}=-1 \\
0 & \text { if } & e^{\prime \prime}=0
\end{array}\right.
$$

After the secret data is extracted, the corresponding prediction error is modified as

$$
e^{\prime}=\left\{\begin{array}{l}
e^{\prime \prime}+1 \text { if } e^{\prime \prime}=-1 \\
e^{\prime \prime} \text { ot her wi se }
\end{array}\right.
$$

Step b-4. Scan $e^{\prime}$ to extract the secret data as

$$
w=\left\{\begin{array}{lll}
1 & \text { if } & e^{\prime}=1 \\
0 & \text { if } & e^{\prime}=0
\end{array}\right.
$$

The corresponding prediction error is update as

$$
e=\left\{\begin{array}{l}
e^{\prime}-1 \text { if } e^{\prime}=1 \\
e^{\prime} \text { if } e^{\prime}=0
\end{array}\right.
$$

Step b-5. Suppose $t$ is a parameter, which is set from 1 to $D L$. If $e^{\prime}$ is $t, w$ is extracted as

$$
\begin{aligned}
& w=\left\{\begin{array}{l}
1 \text { if } e^{\prime}=2 \times t \text { or }-2 \times t \\
0 \text { if } e^{\prime}=2 \times t+1 \text { or }-2 \times t-1
\end{array}\right. \\
& e=\left\{\begin{array}{l}
e^{\prime}-t \text { if } e^{\prime}=2 \times t \\
e^{\prime}-t-1 \text { if } e^{\prime}=2 \times t+1 \\
e^{\prime}+t \quad \text { if } e^{\prime}=-2 \times t \\
e^{\prime}+t+1 \text { if } e^{\prime}=-2 \times t-1
\end{array}\right.
\end{aligned}
$$

The remaining prediction errors are shifted without data extracting as

$$
e=\left\{\begin{array}{l}
e^{\prime}+a+1 \text { if } e^{\prime} \leq-2 \times a-2 \\
e^{\prime}-a-1 \text { if } e^{\prime} \geq 2 \times a+2
\end{array}\right.
$$


Step b-6. Each pixel is reconstructed by

$$
f^{L}(x, y)=\hat{f}^{L}(x, y)-e
$$

Step b-7. Rectangle pixels in the right view are predicted by using rhombus prediction, and operate steps from b-2 to b-6 to restore the rectangle pixels. The right view is completely restored.

Step b-8. Circle pixels in the left view are predicted by using PTE, and steps from b- 2 to b-5 are run to extract the secret data. The rectangle pixels are recovered the same way as recovering the circle pixels in the left view. Finally, $\mathbf{W}$ is completely extracted.
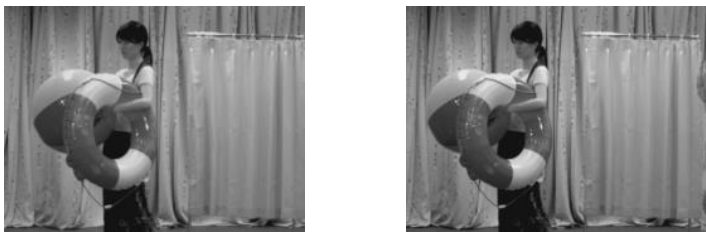

(a)
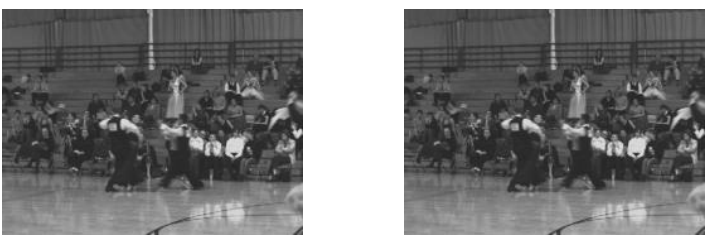

(c)
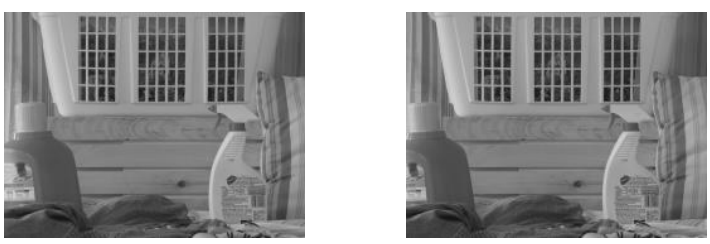

(e)
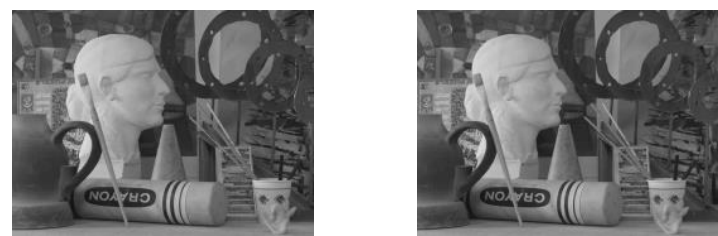

(b)
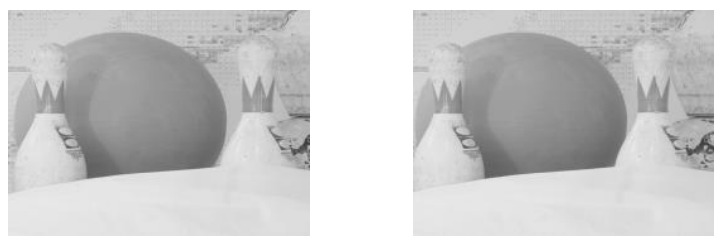

(d)
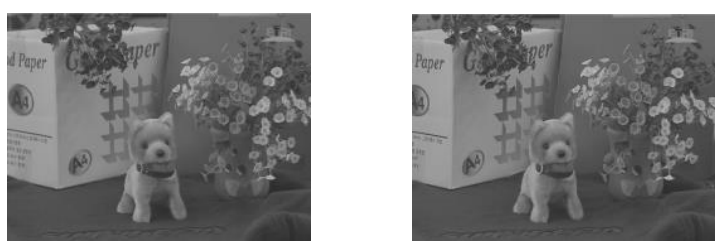

(f)

Fig. 4 Original stereo image. (a) Akko; (b) Art; (c) Ballroom; (d) Bowling; (e) Laundry; (f) Puppy.

\subsection{Prediction Accuracy}

Prediction accuracy of the proposed PTE is tested by comparing with MED, rhombus prediction and DP, where DP predictor is presented in [27]. Fig. 5 shows prediction error histograms of four predictors for circle pattern pixels of left views, and evidently, histograms of PTE are the most sharply distributed compared with other three predictors. It is mainly because inter-correlations of the stereo image increase the accuracy of the prediction. Although DP uses the inter-view relationship as well, prediction accuracy is not high due to matched pixels may not be equal since two views of stereo images are captured by different lighting. Good prediction of PTE leads to good performance of the proposed stereo image reversible data hiding method.

\subsection{Data hiding performance}

When the hiding level $D L$ is set to 0 , the image quality is the best compared with other embedding levels. Table 1 shows the best image quality with different compared reversible data hiding methods, and the proposed method is superior to other three methods in related to the PSNR and embedding capacity. Although PSNRs of Luo's[27] are higher than those of the proposed method, the embedding capacities are much higher compared with Luo's[27]. Thus, it proves that the proposed method is much better than other three methods.

The incensement of $D L$ will lead to high embedding capacity and the low image quality. Since in the proposed method inter-correlation is only used for prediction in the left view but not for the right view, the embedding performance of the left view is compared with different reversible data hiding methods for showing the superiority of the proposed method. 


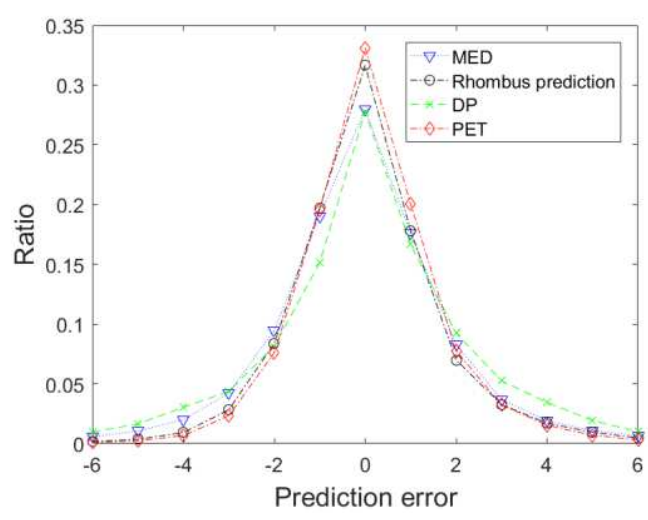

(a)

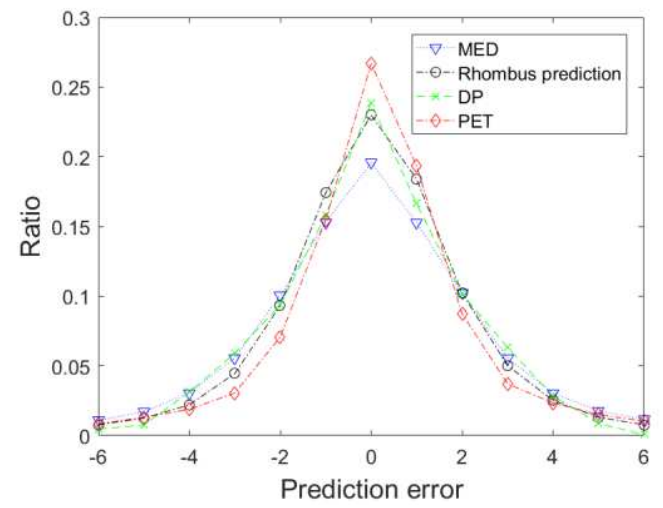

(c)

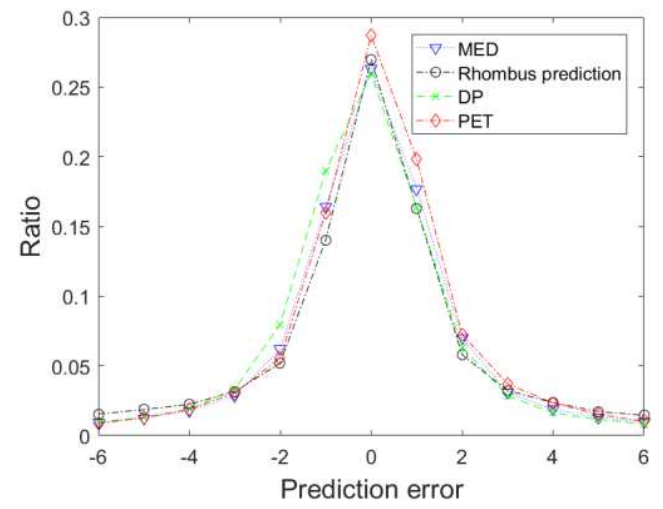

(e)

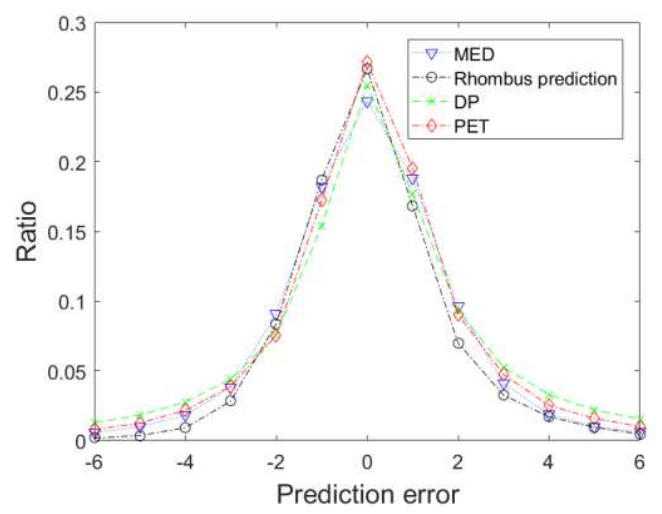

(b)

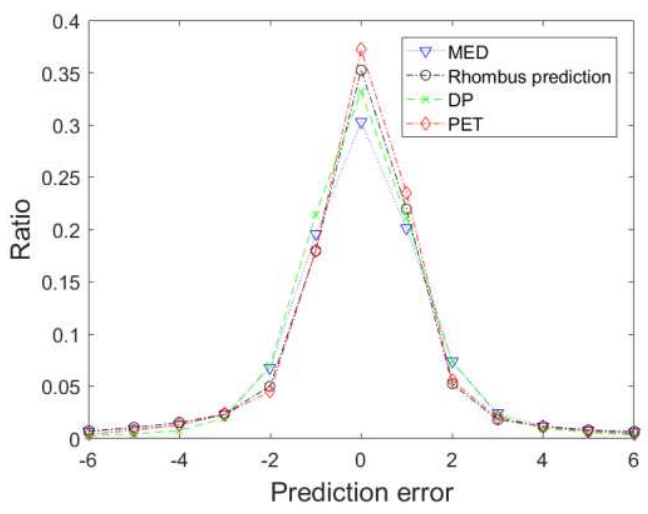

(d)

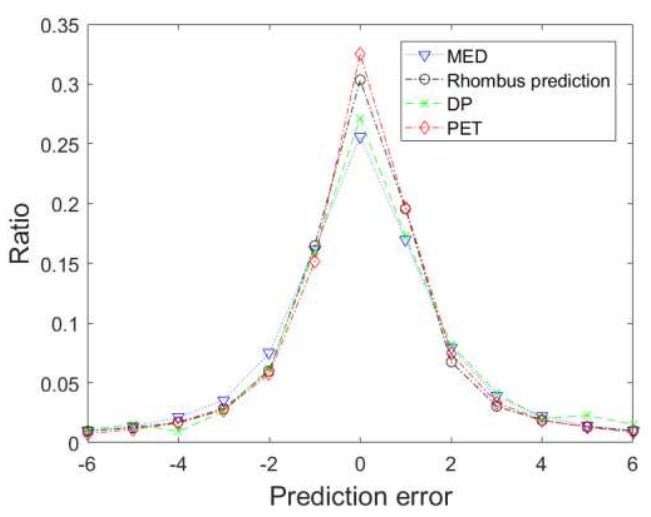

(f)

Fig. 5 Comparison of prediction error histograms. (a) Akko; (b) Art; (c) Ballroom; (d) Bowling; (e) Laundry; (f) Puppy.

Table 1 Image quality and embedding capacity comparison

\begin{tabular}{|c|c|c|c|c|c|c|c|}
\hline & & Akko & Art & Ballroom & Bowling & Laundry & Puppy \\
\hline \multirow{2}{*}{ Kim's[16] } & PSNR[.dB] & 48.98 & 48.97 & 48.89 & 49.25 & 49.06 & 49.09 \\
\hline & Capacity[.bit] & 39782 & 39782 & 29645 & 71424 & 49121 & 53207 \\
\hline \multirow{2}{*}{ Luo’s[17] } & PSNR[.dB] & 49.09 & 49.09 & 48.98 & 49.46 & 49.18 & 49.23 \\
\hline & Capacity[.bit] & 47340 & 47339 & 34560 & 87582 & 57170 & 62967 \\
\hline \multirow{2}{*}{ Luo’s[27] } & PSNR[.dB] & 49.22 & 49.29 & 49.02 & 48.94 & 49.72 & 51.54 \\
\hline & Capacity[.bit] & 80210 & 78582 & 83466 & 132894 & 104110 & 74311 \\
\hline \multirow{2}{*}{ Proposed } & PSNR[.dB] & 49.06 & 49.13 & 48.55 & 48.79 & 49.42 & 52.54 \\
\hline & Capacity[.bit] & 85801 & 84019 & 162939 & 138393 & 110838 & 77384 \\
\hline
\end{tabular}




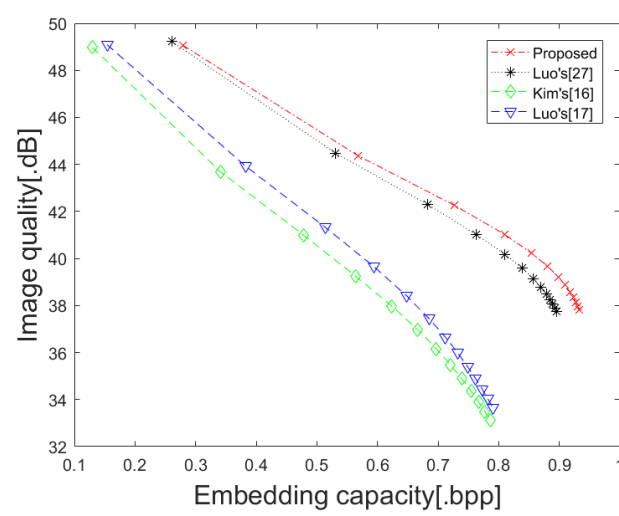

(a)

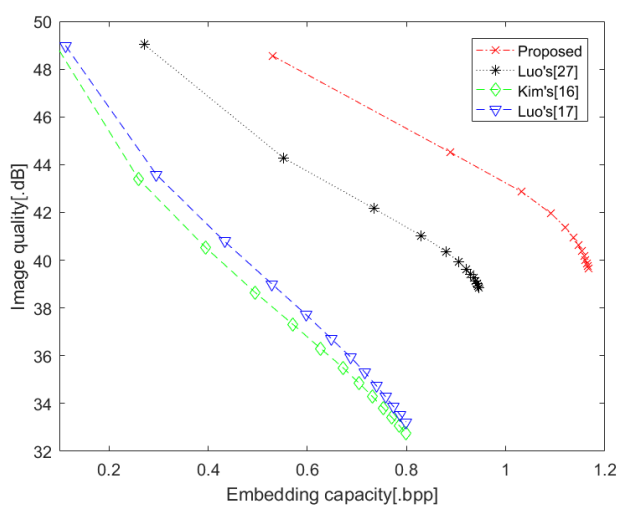

(c)

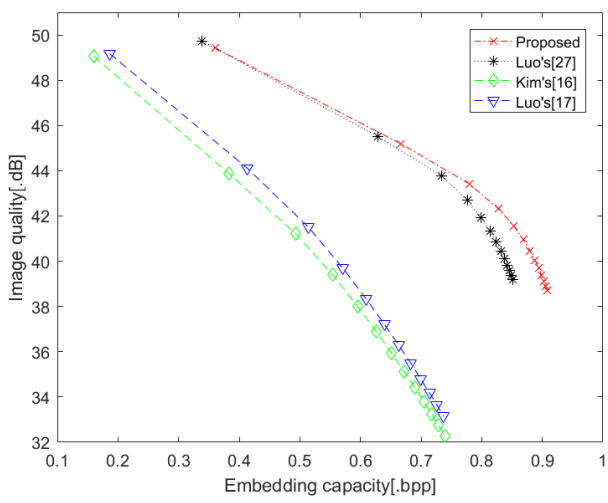

(e)

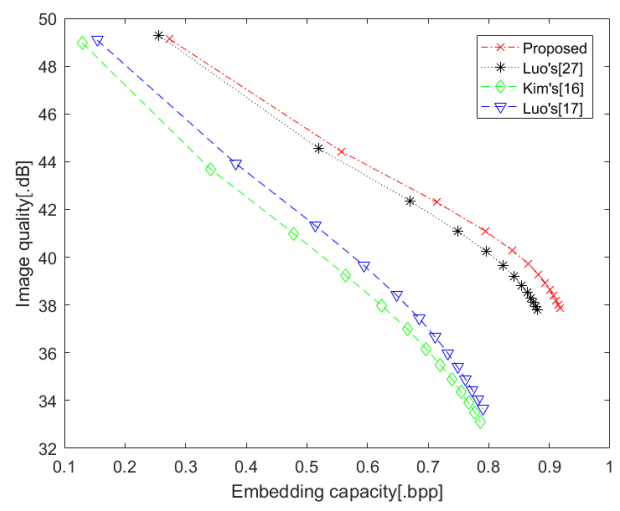

(b)

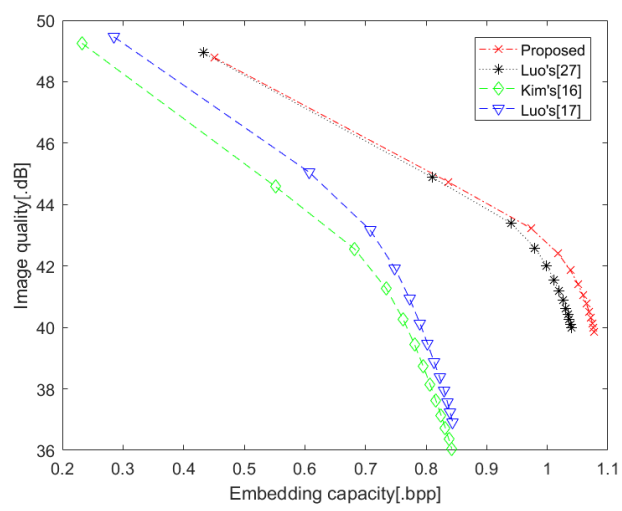

(d)

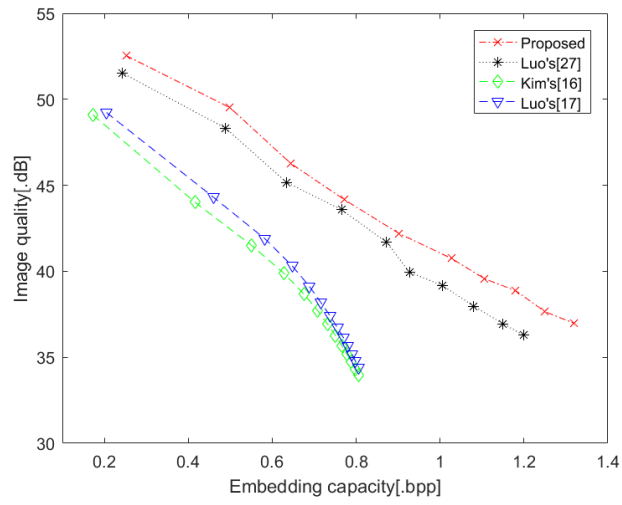

(f)

Fig.6 PSNR versus embedding capacity of the proposed method in comparison with other methods. (a) Akko; (b) Art; (c) Ballroom; (d) Bowling; (e) Laundry; (f) Puppy.

Fig. 6 shows that embedding capacity versus quality for the left view of the proposed method in comparison with other three stereo image reversible data hiding methods. For left views of Ballroom, Bowling and Puppy, when the embedding capacity reach 1 bit per pixel (bpp), and corresponding PSNRs are all higher than $40 \mathrm{~dB}$, which denotes the stego stereo images are cannot be detectable. As $D L$ is increased to more than 4, the embedding capacities are not increased much, but the image is degraded much. The main reason is that prediction errors are mainly around the zero-valued bin. Fig. 6 shows that the proposed method is superior to other methods in related to both of image quality and embedding capacity. PSNRs of the Luo's [27] are lower than those of the proposed method, which denotes that DP is not stable and accurate for predicting all stereo images. Above experiments reveal the effective performance of the proposed method.Experiments are also tested on the Middlebury stereo dataset, and average PSNR and average embedding capacities are calculated. Table 2 shows that the proposed method is much better than Yang's method. It supports that the proposed method achieves high embedding capacity and low image distortion. 
Table 2 PSNR and embedding capacity of the proposed method in comparison with Yang's

\begin{tabular}{llll}
\hline & $E L$ & Quality & PSNR \\
\hline Yang's & N/A & 43.24 & 0.1200 \\
& N/A & 40.98 & 0.2100 \\
Proposed & 0 & 48.50 & 0.4124 \\
& 1 & 44.27 & 0.6321 \\
\hline
\end{tabular}

\section{Conclusion}

In this paper, a novel Taylor Expansion (TE) based stereo image reversible data hiding method is proposed. The accuracy of the prediction is very important for the embedding performance of the stereo image reversible data hiding method, a novel TE based predictor (TEP) is designed by fully using the inter-correlation of the stereo image. Specifically, the matched pixel is searched for predicting the current pixel by computing the second-order TE. Moreover, histogram shifting is utilized to embed data to decrease distortion of stereo images, and multi-level embedding can increase embedding capacity. Experimental results prove that the proposed method is better than existing stereo image reversible data hiding methods in related to the embedding capacity and the stego stereo image quality.

\section{Author's contributions}

YY and GZ conceived and designed the study. GZ performed the experiments. YY wrote the paper. YY and GZ reviewed and edited the manuscript. All authors read and approved the manuscript.

\section{Funding}

This work was supported by Scientific Research Fund of Zhejiang Provincial Education Department Grant(No.Y201430481), and Scientific Research Fund of Ningbo University, Grant(No.XYL12011). It was also sponsored by the K. C. Wong Magna Fund in Ningbo University.

\section{Availability of data and materials}

The data used to support the findings of this study are available from the first author upon request.

\section{Competing interests}

The authors declare that they have no competing interests.

\section{References}

[1] Ntalians, K., Tsapatsoulis N. and Drigas A. Video-Object Oriented Biometrics Hiding for User Authentication under Error-Prone Transmissions. EURASIP Journal on Information Security, 3463,174945 (2011)

[2] Ding X., Raziei Z., Larson E., et al. Swapped face detection using deep learning and subjective assessment. EURASIP Journal on Information Security, 618, 6(2020).

[3] KHAN, Sameeulla; CHANNAPPAYYA, Sumohana S. Estimating Depth-Salient Edges and Its Application to Stereoscopic Image Quality
Assessment. IEEE Transactions on Image Processing, 27(12), 5892-5903(2018).

[4] Luo, T., Jiang, G., Yu, M., \& Xu, H. Asymmetric self-recovery oriented stereo image watermarking method for three dimensional video system. Multimedia Systems, 27(5),641-655(2016).

[5] Luo, T., Jiang, G., Yu, M., Shao, F., Peng, Z., \& Ho, Y. S. Stereo matching based stereo image watermarking for tamper detection and recovery. International Journal of Computational Intelligence Systems, 7(5),874-881(2014).

[6] Luo, T., Jiang, G., Yu, M., Xu, H., \& Gao, W. Sparse recovery based reversible data hiding method using the human visual system. Multimedia Tools and Applications, 77(15),19027-19050(2018).

[7] Dong, K., Kim H., Yu X., et al. Reversible data hiding for binary images based on adaptive overlapping pattern. EURASIP Journal on Information Security, 334, 11(2020).

[8] Fridrich J, Goljan J, Du R. Invertible authentication . In Proceedings of SPIE Security and Watermarking of Multimedia Content III. San Jose, CA, 197-208(2001).

[9] J. Tian, Reversible data embedding using a difference expansion, IEEE Transaction on Circuits and Systems for Video Technology, 13, 890-897(2003).

[10] Alattar, Adnan M. Reversible watermark using the difference expansion of a generalized integer transform. IEEE transactions on image processin. 13(8), 1147-1156(2004).

[11] L. Kamstra, H. Heijmans, Reversible data embedding into images using wavelet techniques and sorting, IEEE Trans. Image Process, 14(12), 2082-2090(2005).

[12] Tang, Z., Xu, S., Yao, H., Qin, C., \& Zhang, X. Reversible data hiding with differential compression in encrypted image. Multimedia Tools and Applications, 78(8), 9691-9715(2019).

[13] Yao, H., Liu, X., Tang, Z., Hu, Y. C., \& Qin, C. An improved image camouflage technique using color difference channel transformation and optimal prediction-error expansion. IEEE Access, 6 , 40569-40584 (2018).

[14] Li, F., Mao, Q., \& Chang, C. C. Reversible data hiding scheme based on the Haar discrete wavelet transform and interleaving prediction method. Multimedia Tools and Applications, 77(5), 5149-5168(2018).

[15] Z. Ni, Y. Q. Shi, N. Ansari, et al., Reversible data hiding, IEEE Transactions on Circuits and Systems for Video Technology, 16, 354-362(2006).

[16] Kim, K. S., Lee, M. J., Lee, H. Y., \& Lee, H. K. Reversible data hiding exploiting spatial correlation between sub-sampled images. Pattern Recognition, 42(11), 3083-3096(2009).

[17] Luo, H., Yu, F. X., Chen, H., Huang, Z. L., Li, H., \& Wang, P. H. (2011). Reversible data hiding based on block median preservation. Information sciences, 181(2), 308-328(2011).

[18] An, L., Gao, X., Yuan, Y., \& Tao, D. Robust lossless data hiding using clustering and statistical quantity histogram. Neurocomputing, 77(1), 1-11(2012).

[19] Kukreja, S., Kasana, S. S., \& Kasana, G. Histogram based multilevel reversible data hiding scheme using simple and absolute difference images. Multimedia Tools and Applications, 78(5), 6139-6162(2019).

[20] Tsai P, Hu Y, Yeh HL.Reversible image hiding scheme using predictive coding and histogram shifting. Signal Process 89(6),1129-1143(2009).

[21] Lu, T. C., Tseng, C. Y., \& Deng, K. M. Reversible data hiding 
using local edge sensing prediction methods and adaptive thresholds. Signal Processing, 104, 152-166(2014).

[22] Ou B, Li X, Zhao Y, et al. Reversible data hiding based on PDE predictor. Journal of Systems and Software, 86(10), 2700-2709(2013).

[23] Yu, M., Wang, J., Jiang, G., Peng, Z., Shao, F., \& Luo, T. New fragile watermarking method for stereo image authentication with localization and recovery. AEU-International Journal of Electronics and Communications, 69(1),361-370(2015)

[24] Zhou, W., Jiang, G., Yu, M., Shao, F., \& Peng, Z. Reduced-reference stereoscopic image quality assessment based on view and disparity zero-watermarks. Signal Processing: Image Communication, 29(1), 167-176(2014)

[25] Wang, C., Wang, X., Xia, Z., \& Zhang, C. Ternary radial harmonic Fourier moments based robust stereo image zero-watermarking algorithm. Information Sciences,470, 109-120(2019).

[26] W. C. Yang, L. H. Chen, Reversible DCT-based data hiding in stereo images. Multimedia Tools and Applications, 74(17), 7181-7193(2015).

[27] T. Luo, G. Jiang, M. Yu, et al., Disparity based stereo image reversible data hiding, 21th IEEE International Conference on Image Processing ,Paris, France, 5492-5496(2014)

[28] Y. Boykov, V. Kolmogorov, An experimental comparison of min-cut/max-flow algorithms for energy minimization in vision. IEEE Transactions on Pattern Analysis and Machine Intelligence, 26(9), 1124-1137(2014). 
Figures

\begin{tabular}{|c|c|c|}
\hline $\begin{array}{l}(x-1, \\
y-1)\end{array}$ & $(x-1, y)$ & $\begin{array}{l}(x-1, \\
y+1)\end{array}$ \\
\hline$(x, y-1)$ & $(x ; y)$ & $(x, y+1)$ \\
\hline $\begin{array}{l}(x+1, \\
y-1)\end{array}$ & $(x+1, y)$ & $\begin{array}{l}(x+1, \\
y+1)\end{array}$ \\
\hline
\end{tabular}

Figure 1

Location of neighbor pixels

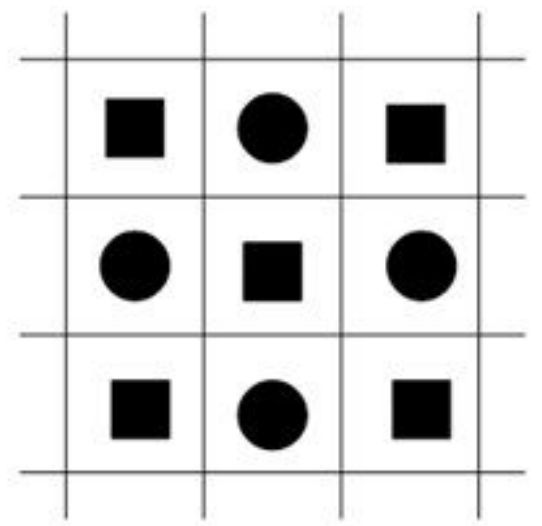

Figure 2

Pixel pattern

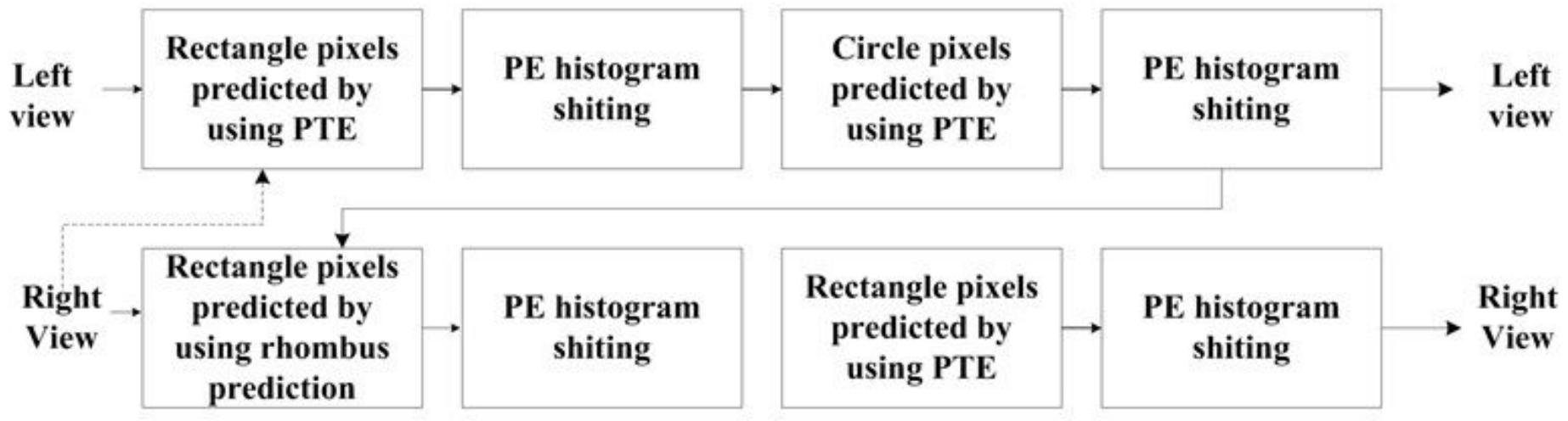

Figure 3 
Block diagram of data embedding
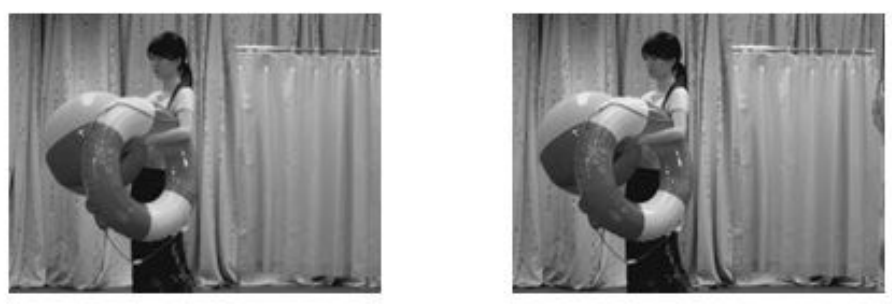

(a)
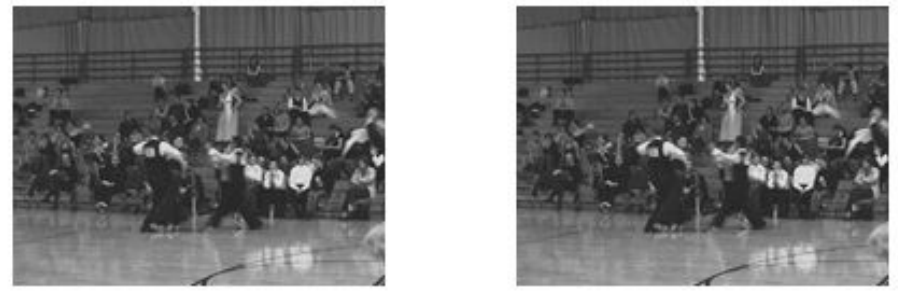

(c)

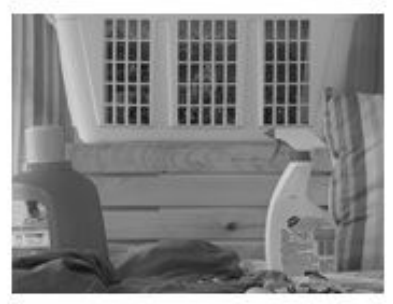

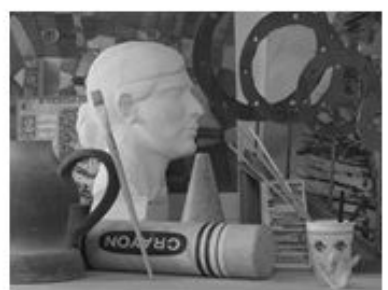

(b)

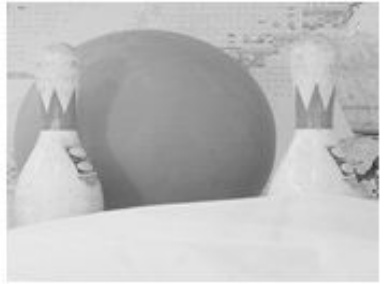

(d)

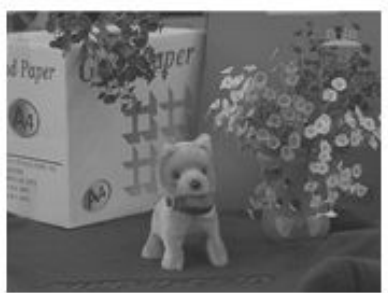

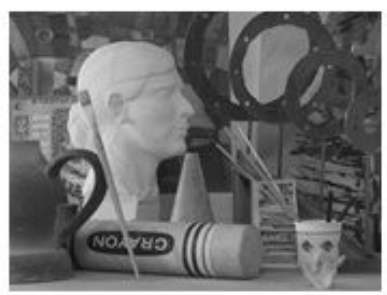
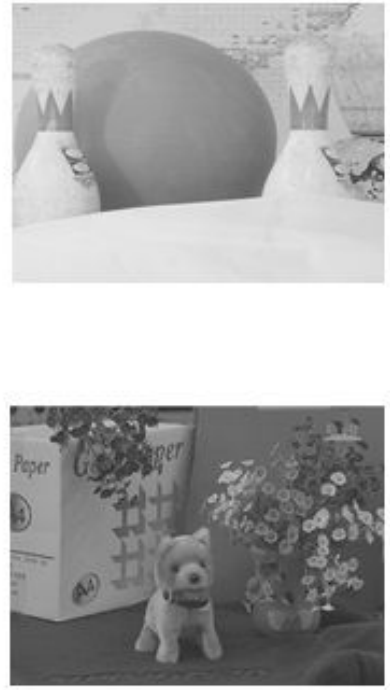

(f)

(e)

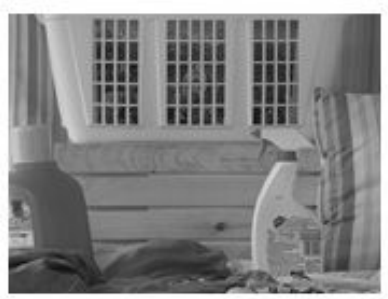

Figure 4

Original stereo image. (a) Akko; (b) Art; (c) Ballroom; (d) Bowling; (e) Laundry; (f) Puppy 


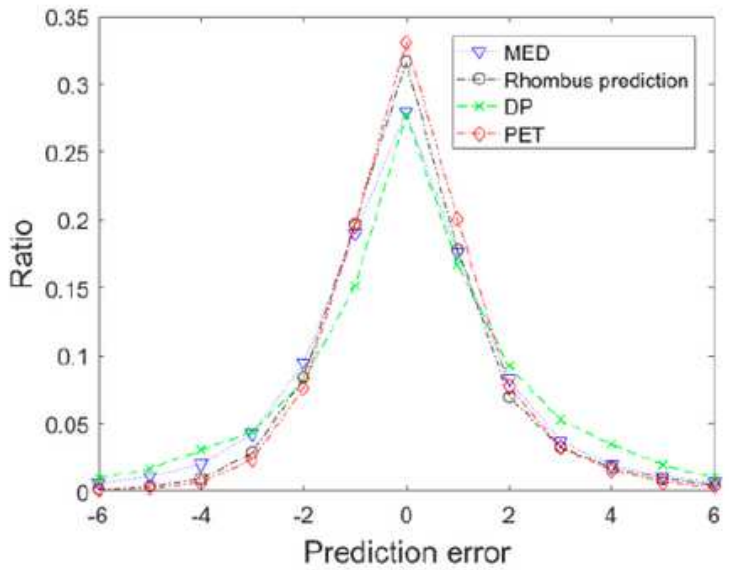

(a)

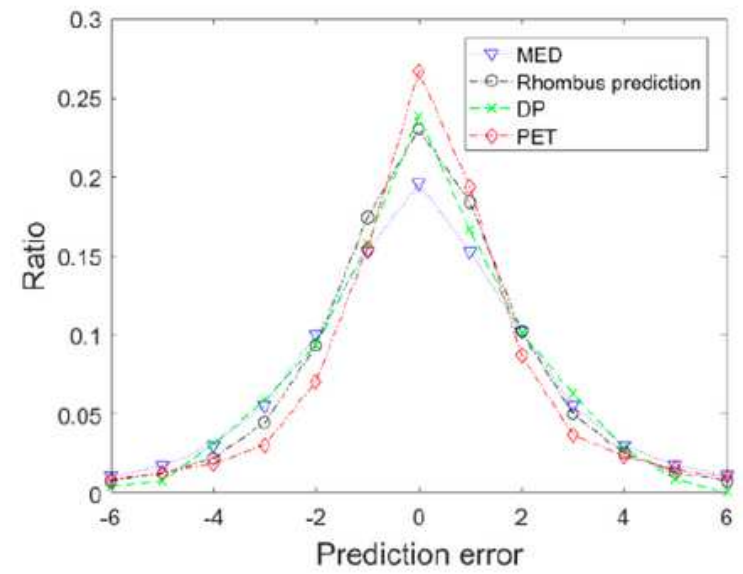

(c)

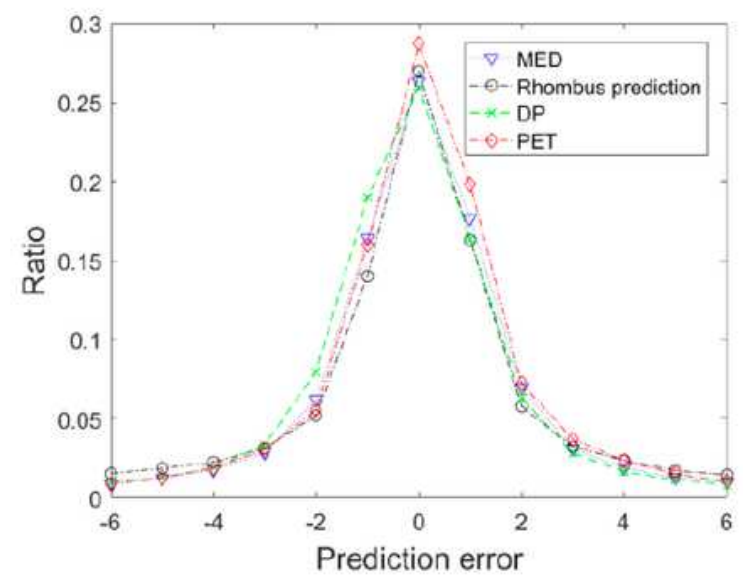

(e)

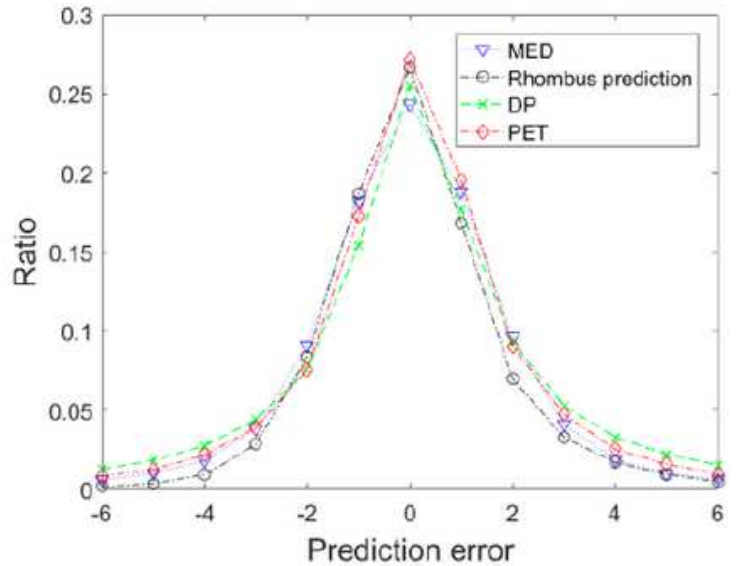

(b)

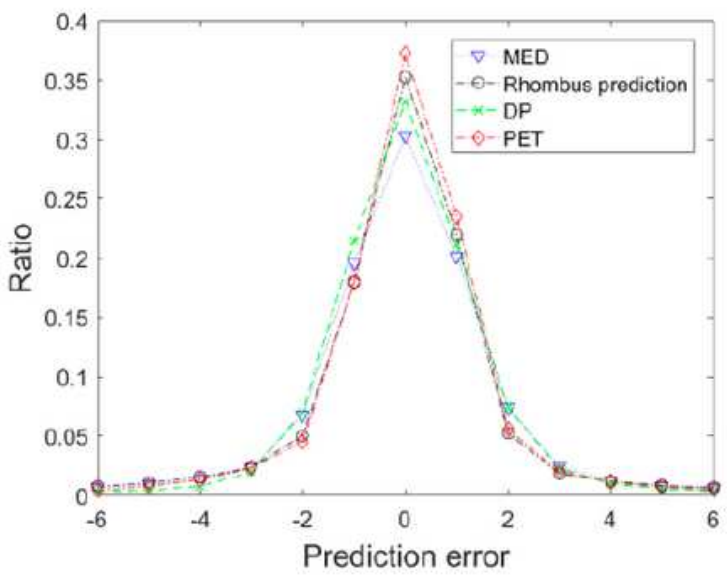

(d)

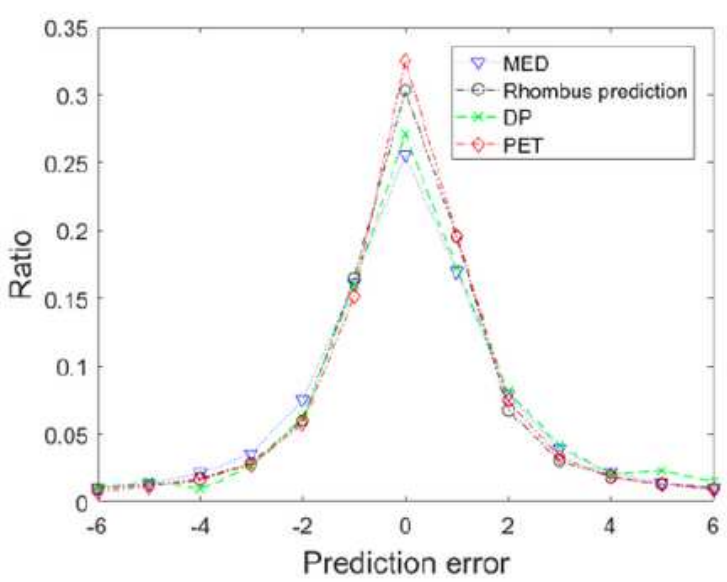

(f)

\section{Figure 5}

Comparison of prediction error histograms. (a) Akko; (b) Art; (c) Ballroom; (d) Bowling; (e) Laundry; (f) Puppy. 


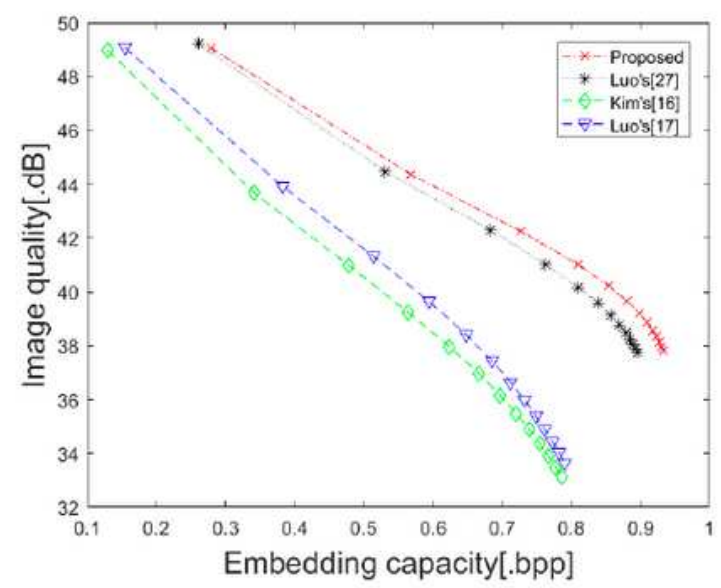

(a)

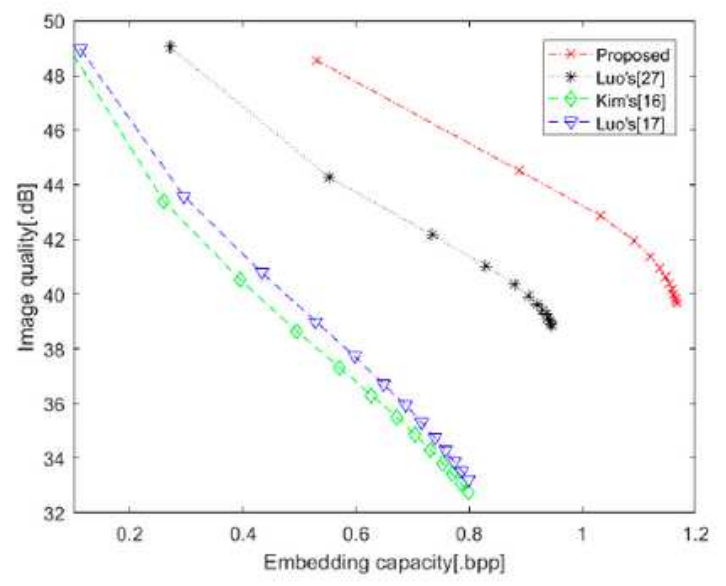

(c)

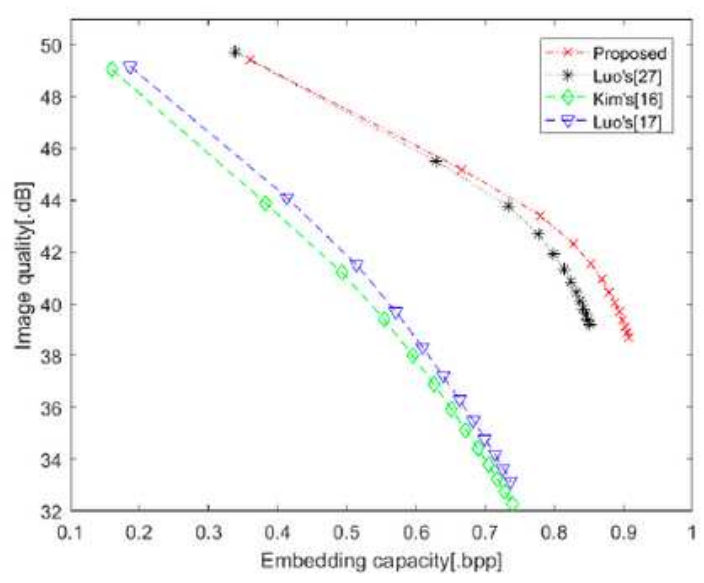

(e)

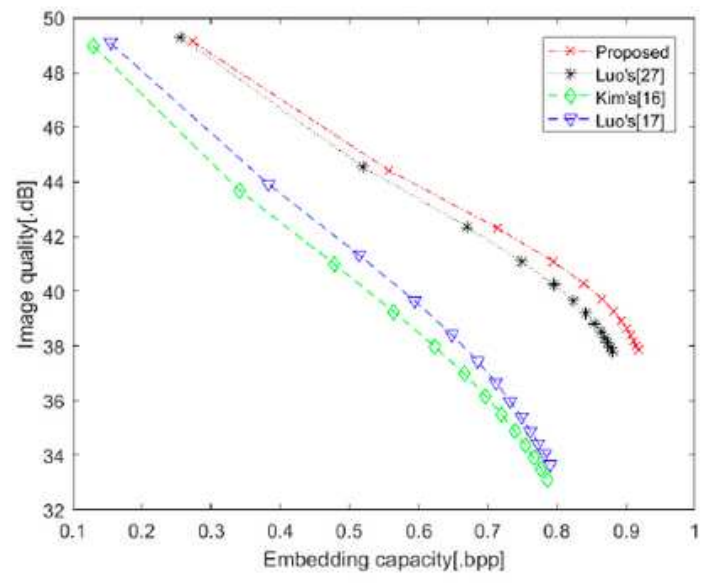

(b)

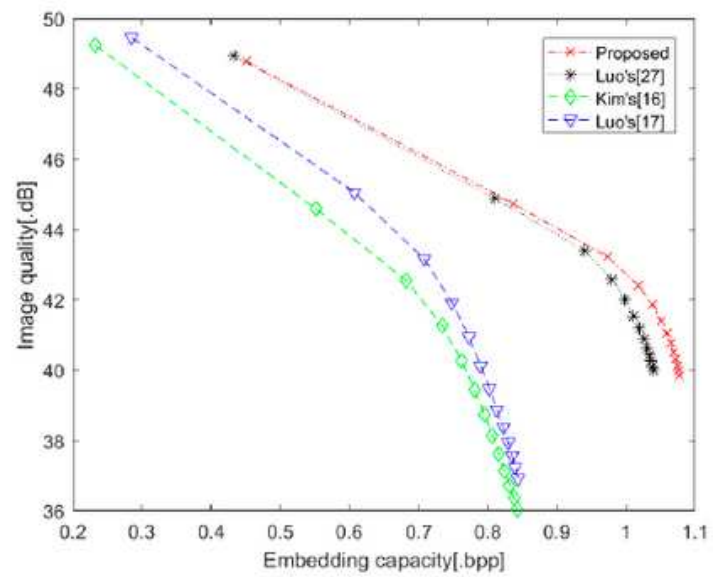

(d)

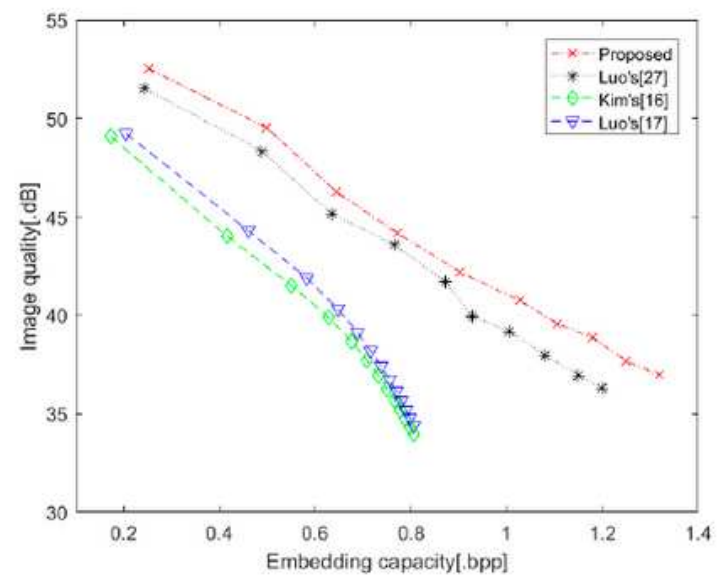

(f)

\section{Figure 6}

PSNR versus embedding capacity of the proposed method in comparison with other methods. (a) Akko; (b) Art; (c) Ballroom; (d) Bowling; (e) Laundry; (f) Puppy. 